\title{
Pose Insensitive 3D Retrieval by Poisson Shape Histogram
}

\author{
Pan Xiang ${ }^{1}$, Chen Qi Hua ${ }^{2}$, Fang Xin Gang ${ }^{1}$, and Zheng Bo Chuan ${ }^{3}$ \\ ${ }^{1}$ Institute of Software, Zhejiang University of Technology \\ ${ }^{2}$ Institute of Mechanical, Zhejiang University of Technology \\ ${ }^{3}$ College of Mathematics \& Information, China West Normal University \\ 1,2310014, Zhejiang, ${ }^{3} 637002$, Nanchong, P.R. China \\ panxiangideyahoo.com
}

\begin{abstract}
With the rapid increase of available 3D models, content-based 3D retrieval is attracting more and more research interests. Histogram is the most widely in constructing $3 \mathrm{~d}$ shape descriptor. Most existing histogram based descriptors, however, will not remain invariant under rigid transform. In this paper, we proposed a new kind of descriptor called poisson shape histogram. The main advantage of the proposed descriptor is not sensitive for rigid transform. It can remain invariant under rotation as well. To extract poisson shape histogram, we first convert the given $3 \mathrm{~d}$ model into voxel representation. Then, the poisson solver with dirichlet boundary condition is used to get shape signature for each voxel. Finally, the poisson shape histogram is constructed by shape signatures. Retrieving experiments for the shape benchmark database have proven that poisson shape histogram can achieve better performance than other similar histogram-based shape representations.
\end{abstract}

Keywords: 3D shape matching, Pose-Insensitive, Poisson equation, Histogram.

\section{Introduction}

Recent development in modeling and digitizing techniques has led to a rapid increase of 3D models. More and more 3D digital models can be accessed freely from Internet or from other resources. Users can save the design time by reusing existing 3D models. As a consequence, the concept has changed from "How do we generate 3D models?" to "How do we find them?"[1]. An urgent problem right now is how to help people find their desirable 3D models accurately and efficiently from the model databases or from the web. Content-based 3D retrieval aiming to retrieve $3 \mathrm{D}$ models by shape matching has become a hot research topic.

In Content-based 3D retrieval, histogram based representation has been widely used for constructing shape features[2]. For histogram based representation, it needs to define shape signatures. The defined shape signature is the most important for histogram descriptor. It should be invariant to affine transformations such as translation, scaling, rotation and rigid transform. Some rotation invariant shape signatures, such as curvature, distance et al, have been used for content-based $3 \mathrm{~d}$ retrieval. Those 
shape signatures are independent of $3 \mathrm{~d}$ shape rotation. However, little researches are focusing on extracting invariant shape signatures under rigid transform. Those existing rotation-invariant shape signatures are often sensitive to rigid transform.

In this paper, we propose a new kind of shape signature called poisson shape measure. It can remain almost invariant under not only rotation transform, but also rigid transform. The proposed shape signature is based on poisson theory. As one of the most important PDE theory, it has been widely used for computer vision, computer graphics, analysis of anatomical structures and image processing[3-5]. However, it has not been used for defining $3 \mathrm{~d}$ shape signature and then content based $3 \mathrm{~d}$ retrieval. The process of constructing poisson shape histogram can be concluded as following: the given $3 \mathrm{~d}$ model will be first converted into voxel representation. Then, the poisson solver with dirichlet boundary condition is used to get shape signature for each voxel. Finally, the poisson shape histogram is constructed by the shape signatures. The comparative study shows poisson shape histogram can achieve better retrieving performance than other similar histogram descriptors.

The remainder of the paper is organized as follows: Section 2 provides a brief review of the related work. Section 3 discusses the poison equation and the related property. Section 4 discusses how to construct poisson shape histogram. Section 5 provides the experimental results for content-based 3D retrievals. Finally, Section 6 concludes the paper and recommends some future work.

\section{Related Work}

Previous shape descriptors can be classified into two groups by their characteristics: namely structural representation and statistical representation. The method proposed in this paper belongs to statistical representation. This section mainly gives a brief review on statistical shape description for content-based 3D retrieval. For more details about structure descriptors and content-based 3D retrieval, please refer to some survey papers[6-8].

As for statistical representation, the most common approach is to compute geometry signatures of the given model first, such as normal, curvature, distance and so on. Then, the extracted shape signatures are used to construct histogram. Existing shape signatures for $3 \mathrm{~d}$ shape retrieval can be grouped into two types: one is the rotation invariant shape signatures, and the other is not. For the latter, rotation normalization is performed prior to the extraction of shape signatures.

\section{Rotation variant shape signatures}

Extend Gaussian Image (EGI) defines shape feature by normal distribution over the sphere[9]. An extension version of EGI is the Complex Extend Gaussian Image (CEGI)[10], which combines distance and normal for shape descriptor. Shape histograms defined on shells and sectors around a model centroid is to capture point distribution[11]. Transform-based shape features can be seen as a post-process of the original shape signatures. It often can achieve better retrieving accuracy than the 
original shape signatures. Vranic et al perform spherical harmonics transform for point distribution of the given model[12]. While Chen et al considered the concept that two models are similar if they look similar from different view angles. Hence they extracted transform coefficients in 2D spaces instead of the 3D space[13]. Transform-based 3D retrievals often can achieve better retrieving performance than histogram-based methods. but are more computational costly.

\section{Rotation invariant shape signatures}

This kind of shape signature is robust again rotation transform. Shape distribution used some measures over the surfaces, such as distance, angle and area, to generate histograms[14]. The angle and distance distribution (AD) is to integrate normal information into distance distribution[15]. The generalized shape distributions is to combine local and global shape feature for $3 \mathrm{~d}$ retrieval. Shape index defined by curvature is adopted as MPEG-7 3D shape descriptor[16]. Radius-Angle Histogram is to extract the angle between radius and normal for histogram[17]. The local diameter shape-function is to compute the distance from surface to medial axis[18]. It has the similar characteristic with the poisson measure proposed by this paper. The extraction of local diameter shape function, however, is very time-cost(It requires nearly 2 minutes in average for construing histogram).

\section{Poisson Equation}

Poisson's equation arises in gravitation and electrostatics, and is the fundamental of mathematical physics. Mathematically, Poisson's equation is a second-order elliptic partial differential equation defined as:

$$
\Delta U=-1
$$

where $\Delta U$ is the laplacian operation. The poisson equation is to assign every internal point a value. As for definition, the poisson equation is somewhat similar with distance transform. The distance transform will assign to every internal point a value that depends on the relative position of that point within the given shape, which reflects its minimal distance to the boundary. The poisson equation, however, has a huge difference with distance transform. The poisson is to place a set of particles at the point and let them move in a random walk until they hit the contour. It measures the mean time required for a particle to hit the boundaries. That's to say, the poisson equation will consider each internal point affected one more boundary points, and will be more robust again distance transform.

The poisson equation has the potential property in shape analysis. Here we show some of these properties.

1. Rotation invariant. Poisson equation is independent of the coordinate system over the entire domain (volume in $3 \mathrm{D}$, and region in $2 \mathrm{D}$ ). It makes the signature defined by poisson equation be robust again rotation.

2. Geometry structure related. The poisson equation is correlated to the geometry of the structure. This correlation gives a mathematical meaning to the shape structure. 
3. Rigid-transform invariant. Similar with geodesic distance, the poisson equation has a strong robustness over the rigid transform.

\section{Poisson Shape Histogram and Matching}

Followed by the definition of poisson equation, this section will discuss how to construct poisson shape histogram and similarity calculation.

The definition of poisson equation is to assign each internal point a value. Most 3D models, however, will use boundary representation, such as the mesh model. The given mesh model will be converted into $3 \mathrm{~d}$ discrete grid $(48 \times 48 \times 48)$ first. The voxelization algorithm used in this paper is based on Z-buffer[19]. The efficiency of this algorithm is independent of the object complexity, and can be implemented efficiently. The voxelization also make a process of scale normalization for the given model.

Suppose the voxelization model can be represented by a finite voxel set $V_{i}, i=1,2, \cdots \cdots N$, where $\mathrm{N}$ is total voxel count. The tacus package[20] is then used for poisson solver. After that, for each voxel $V_{i}$, we can get poisson shape signature, denoted by $P_{i}$. The construction of poisson shape histogram can be concluded as the following steps:

1) For the signature set $P_{i}, i=1,2, \cdots i, \cdots N$, compute its mean value $\mu$ and variance $\sigma$ respectively.

2) For each $P_{i}$, perform Gaussian normalization by the following equation.

$$
P_{i}^{\prime}=\frac{P_{i}-\mu}{3 \sigma}
$$

3) For normalized set $P_{i}^{\prime}$, construct histogram containing 20 bins, denoted by:

$$
H=\left\{H_{1}, H_{2}, \cdots H_{i}, \cdots H_{20}\right\}
$$

For two histograms, we use $\mathrm{L}_{1}$-metric to measure their dissimilarity.

$$
\operatorname{Dis}_{1,2}=\sum\left|H_{1, i}-H_{2, i}\right|, i=1,2, \cdots \cdots N \text {. }
$$

where $\mathrm{H}_{1}$ and $\mathrm{H}_{2}$ denote poisson shape histogram for two models. The bigger value means two models are more dissimilar.

Section 3 discusses the property of poisson equation, and it shows the poisson equation will be independent of rigid transform. Figure 1 gives poisson shape histogram for horses under different rigid transform. The poisson shape histogram remains almost invariant for different rigid transform(the small difference due to the voxelization error). As a comparison, the D2 shape distribution, however, appears to be huge difference for two models. 


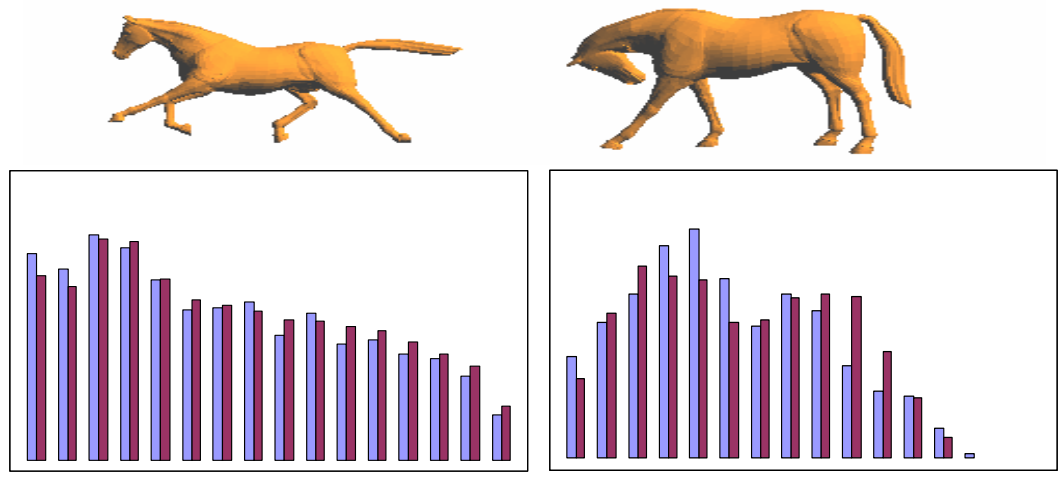

Fig. 1. Histogram descriptors for above models(Upper: Horses under different rigid-transform. Lower: The left is the poisson shape histogram for the above two models, and the right is the D2 shape distribution as well. The difference between poisson shape histograms appear to be very minor. While the difference of the D2 shape distributions appears to be very obvious).

\section{Experiment}

Experiments are carried out to test the retrieving performance of poisson shape histogram. All experiments are performed with the hardware Intel Pentium 1.86GHZ, 512M memory. The testing 3D models are Princeton Shape Benchmark database(PSB)[21]. It contains 1804 mesh models, and is classified into two groups. Each group contains 907 models as well. One is the training set, which is used to get best retrieving parameters. The other is the testing set for retrieving performance comparison of different shape descriptors. The benchmark also provides different evaluating criterions for retrieving precision. Here we use Precision-Recall curve to measure the retrieving accuracy, and the precision-recall measure has been widely used in information retrieval. We first show the time in constructing shape poisson histogram, and then retrieving accuracy comparison with similar histograms.

As for content-based 3D retrieval, the feature extraction process should be performed quickly. This is very important, especially for practical applications. The costing time for building poisson shape histogram consists of the following steps: voxelization, poisson solver and histogram construction. The voxelization time is almost $0.07 \mathrm{~s}$ for each model, and the histogram construction is near to 0s. Notice the time for poisson solver is related with the count of voxel. Table 1 shows the costing time for different voxel models.

In average, the costing time for poisson shape histogram is about $0.6 \mathrm{~s}$. While for D2 shape distribution, the generating time is about $0.8 \mathrm{~s}$.

Next, we will compare the retrieving performance of poisson shape histo$\operatorname{gram}(\mathrm{PSH})$ with some other histogram based shape descriptors. They are 3D shape spectrum(3DS), and D2 distance(D2). Figure 2 givens the precision-recall curve for 
Table 1. The costing time for poisson solver

\begin{tabular}{|c|c|}
\hline Voxel models & Poisson solver (s) \\
\hline 8624 & 1.1 \\
\hline 6832 & 0.7 \\
\hline 4500 & 0.4 \\
\hline 2306 & 0.2 \\
\hline
\end{tabular}

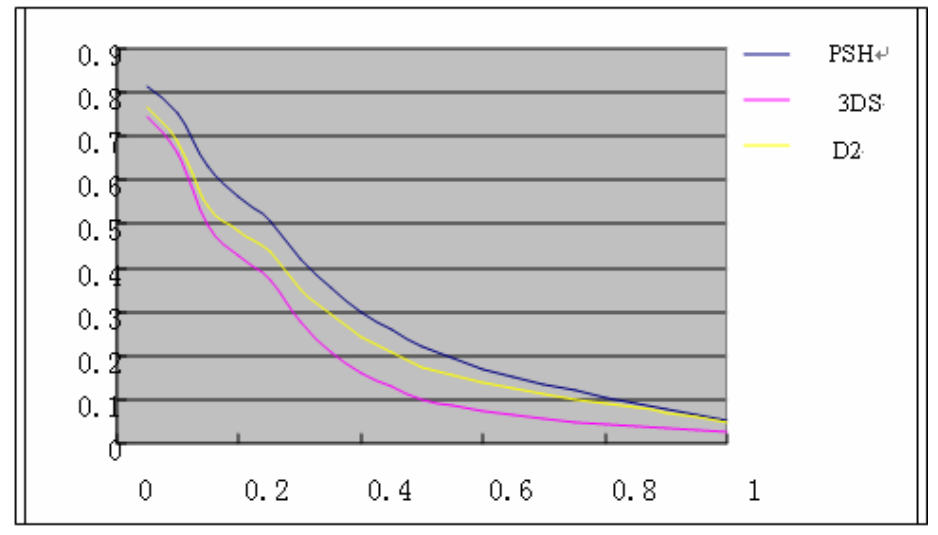

Fig. 2. The Precision-Recall curves for different histogram-based descriptors
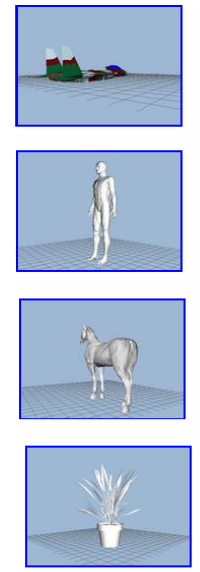
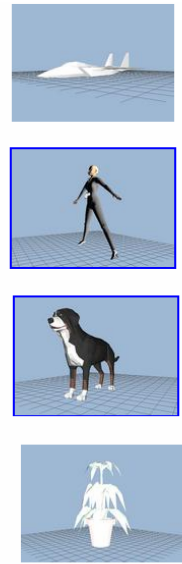
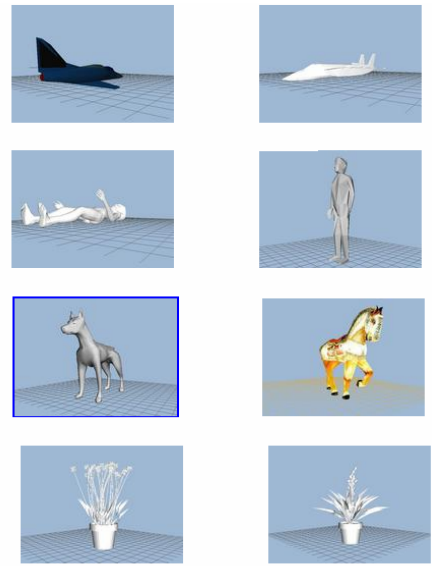

Fig. 3. Some retrieving results(For each row, the left model is the query model, and other three models are the most similar with queried model. Notice the model under different rigid transform can be retrieved correctly). 
three kinds of shape descriptors. It shows the poisson shape histogram can achieve best retrieving precision. Some retrieving results are also shown in Figure 3. Notice those models under different rigid transform can be retrieved correctly.

\section{Conclusion and Future Work}

This paper proposed a new kind of $3 \mathrm{~d}$ shape descriptor called poisson shape histogram. It uses poisson equation as the main mathematical theory. The encouraging characteristic of poisson shape histogram is insensitive for rigid transform. It remains rotation invariant as well. The retrieving experiments have shown that the poisson shape histogram can achieve better retrieving precision than other similar histogrambased 3d shape descriptors.

As a kind of histogram, the main drawback of poisson shape histogram can only capture global shape feature. It can not support partial matching. While for the definition of poisson equation, the poisson shape signature is only affected by local neighbors. It shows the poisson shape measure can represent local shape feature as well. As one of the future work, we will work for partial matching based on poisson equation.

Acknowledgments. This work was supported by natural science foundation of Zhejiang Province(Grant No: Y106203, Y106329). It was also partially funded by the Education Office of Zhejiang Province(Grant No 20051419) and the Education Office of Sichuan Province(Grant No 2006B040).

\section{References}

1. T. Funkhouser, P. Min, and M. Kazhdan, A Search Engine for 3D Models. ACM Transactions on Graphics, (2003)(1): 83-105.

2. Ceyhun Burak Akgül, Bülent Sankur, Yücel Yemez, et al., A Framework for HistogramInduced 3D Descriptors. European Signal Processing Conference (2006).

3. L. Gorelick, M. Galun, and E. Sharon Shape representation and classification using the poisson equation. CVPR, (2004): 61-67.

4. Y. Yu, K. Zhou, and D. Xu, Mesh Editing with Poisson-Based Gradient Field Manipulation. ACM SIGGRAPH, (2005).

5. H. Haider, S. Bouix, and J. J. Levitt, Charaterizing the Shape of Anatomical Structures with Poisson's Equation. IEEE Transactions on Medical Imaging, (2006). 25(10): 1249-1257.

6. J. Tangelder and R. Veltkamp. A Survey of Content Based 3D Shape Retrieval Methods. in International Conference on Shape Modeling. (2004).

7. N. Iyer, Y. Kalyanaraman, and K. Lou. A reconfigurable $3 D$ engineering shape search system Part I: shape representation. in CDROM Proc. of ASME 2003. (2003). Chicago.

8. Benjamin Bustos, Daniel Keim, Dietmar Saupe, et al., An experimental effectiveness comparison of methods for $3 D$ similarity search. International Journal on Digital Libraries, (2005).

9. B. Horn, Extended Gaussian Images. Proceeding of the IEEE, (1984). 72(12): 1671-1686. 
10. S. Kang and K. Ikeuchi. Determining 3-D Object Pose Using The Complex Extended Gaussian Image. in International Conference on Computer Vision and Pattern Recognition. (1991).

11. M. Ankerst, G. Kastenmuller, H. P. Kriegel, et al. 3D Shape Histograms for Similarity Search and Classification in Spatial Databases. in International Symposium on Spatial Databases. (1999).

12. D. Vranic, 3D Model Retrieval, PH. D Thesis, . 2004, University of Leipzig.

13. D. Y. Chen, X. P. Tian, and Y. T. Shen, On Visual Similarity Based 3D Model Retrieval. Computer Graphics Forum (EUROGRAPHICS'03), (2003). 22(3): 223-232.

14. R. Osada, T. Funkhouser, B. Chazelle, et al. Matching 3D Models with Shape Distributions. in International Conference on Shape Modeling and Applications. (2001).

15. R. Ohbuchi, T. Minamitani, and T. Takei. Shape-Similarity Search of 3D Models by Using Enhanced Shape Functions. in Theory and Practice of Computer Graphics. (2003).

16. T. Zaharia and F. Preteux. 3D Shape-based Retrieval within the MPEG-7 Framework. in SPIE Conference on Nonlinear Image Processing and Pattern Analysis. (2001).

17. Xiang Pan, Yin Zhang, Sanyuan Zhang, et al., Radius-Normal Histogram and Hybrid Strategy for 3D Shape Retrieval. International Conference on Shape Modeling and Applications, (2005): 374-379.

18. Ran Gal, Ariel Shamir, and Daniel Cohen-Or, Pose Oblivious Shape Signature. IEEE Transactions of Visualization and Computer Graphics, (2005).

19. E. A. Karabassi, G. Papaioannou, and T. Theoharis, A Fast Depth-buffer-based Voxelization Algorithm. Journal of Graphics Tools, (1999). 4(4): 5-10.

20. S. Toledo, TAUCS: A Library of Sparse Linear Solvers. Tel-Aviv University, 2003. http://www.tau.ac.il/ stoledo/taucs.

21. P. Shilane, K. Michael, M. Patrick, et al. The Princeton Shape Benchmark. in International Conference on Shape Modeling. (2004). 